INVESTIGACIÓN

\title{
CARACTERIZACION REPRODUCTIVA DE LA ESPECIE Prochilodus reticulatus COMO POTENCIAL PRODUCTIVO PARA LA ZONA DEL CATATUMBO- NORTE DE SANTANDER
}

\section{REPRODUCTIVE CARATERIZATION OF THE $P$. reticulatus SPECIE AS A PRODUCTIVE POTENCIAL IN THE CATATUMBO NORTE DE SANTANDER AREA}

\author{
MSc.Carmen Liceth Garcia Quintero ${ }^{\text {a }}$ \\ ${ }^{a}$ Universidad Francisco de Paula Santander Ocaña, GI@DS. Cra 32 N0. 1ª-40 Ocaña Norte de \\ Santander, Colombia, clgarciaq@ufpso.edu.co
}

Fecha de recepción: 03-06-2015

Fecha de aprobación: 021-09-2016

\begin{abstract}
Resumen: La evaluación reproductiva y la caracterización biológica y morfométrica de la especie Prochilodus reticulatus, endémica del rio Catatumbo como prioridad en los programas de investigación y extensión de la Universidad Francisco de Paula Santander Ocaña, permitió establecer programas de repoblamiento, extensión y asistencia técnica para los piscicultores en la zona del Catatumbo.
\end{abstract}

Palabras clave: Bocachico, producción, protocolos, reproducción.

Abstract: Reproductive, biological and morphometric characterization of the species Prochilodus reticulatus, endemic Catatumbo River as a priority in research programs and extension of Francisco de Paula Santander University Ocaña, allowed establishing resettlement programs, technical assistance in production for farmers in the Catatumbo.

Keywords: Bocachico, production, protocols, reproduction. 


\section{INTRODUCCIÓN}

El orden Characiforme, domina las aguas dulces continentales suramericanas; diversificado en multitud de formas que ocupan un amplio rango de nichos ecológicos, su éxito adaptativo, se debe a la diversidad morfológica y fisiológica de sus especies como respuesta a las exigencias propias de cada tipo de ecosistemas. El alto grado de especiación en aguas suramericanas, (más de 1.000 especies), lo constituyen en el grupo de mayor importancia desde el punto de vista ecológico y económico. La importancia del Orden Characiforme como fuente de alimentación para los habitantes de las zonas ribereñas de nuestras cuencas, radica en que en él están incluidas especies destacadas como:

Prochilodus spp. (Bocachico)

Potamorhina laticeps (Manamana)

Mylossoma acanthogaster (Pampano)

Cyrtocharax magdalenae (Mueluda).

En la cuenca del Catatumbo, las dos primeras constituyen el $70 \%$ de la captura. Prochilodus es el género cuyas especies aportan los más altos índices de captura en las zonas de pesca de los ríos Magdalena y Catatumbo.

Antes del levantamiento de los Andes, los ríos Magdalena, Orinoco y Catatumbo confluían en el Lago de Maracaibo, compartiendo la ictiofauna. El levantamiento de la cordillera, aisló dichas cuencas, gestando un proceso de adaptación a las nuevas condiciones y propiciando especialización y especiación. Posteriormente, el levantamiento de la Serranía del Perija, aisló el rio Magdalena del rio Catatumbo, Schultz (1949) citado por Taphor 1992; aunque algunos autores han clasificado al bocachico del Catatumbo como una subespecie, otros niegan que haya especiación, sin embargo las características de los dos grupos presentan diferencias fenotípicas $\quad y$ aun comportamientos reproductivos diferentes.

El género Prochilodus, incluye especies migratorias endémicas para Colombia de amplia distribución en los sistemas de los ríos Sinú, Magdalena, Atrato y Catatumbo.

Para el río Magdalena se encuentra la especie $\boldsymbol{P}$. magdalenae y para el Catatumbo la especie $\boldsymbol{P}$. reticulatus, estas especies presentan alimentación limnofaga que incluye diatomeas y otros organismos del zooplancton bentonico.

Los bocachicos, realizan migraciones reproductivas, por ello se denominan especies reofilas. La maduración gonadal está influenciada por condiciones físicoquímicas del agua y el paso de un ecosistema lentico, las ciénagas, a un ecosistema lotico, el rio, ofrece las condiciones óptimas para su maduración gonadal y finalmente el desove, el cual sucede aguas arriba del rio. Los huevos una vez fertilizados, son arrastrados agua abajo, las larvas eclosionan ingresando a través de los caños de nuevo a las ciénagas, esto hace que la reproducción en cautiverio solo se pueda hacer mediante la inducción hormonal de los reproductores. 
La Universidad Francisco de Paula Santander, ha orientado sus esfuerzos de investigación en la estandarización de las técnicas de reproducción de especies comerciales y nativas en la cuenca del rio Catatumbo, como resultados de estos trabajos en los últimos años, se logra estandarizar la reproducción inducida de varias especies con productos convencionales como Extracto de hipófisis de carpa EPC.

La evaluación reproductiva de la especie Prochilodus reticulatus, endémica del rio Catatumbo, ha sido prioridad en los programas de investigación y extensión de la Universidad Francisco de Paula Santander Ocaña, pues el repoblamiento de esta especie fue compromiso institucional con las comunidades de la zona de influencia de la estación piscícola de San Pablo.

En el presente trabajo, se describe la biología de la especie $P$. reticulatus, se establece la morfometria y merística de la especie $P$. reticulatus, asi mismo se definen $\mathrm{y}$ establecen protocolos reproductivos en cautiverio de la especie en la Estación piscícola de San Pablo Teorama Norte de Santander.

\section{METODOLOGÍA}

El plantel de reproductores está conformado por un total de 248 ejemplares con pesos promedios registrados de 341 gr para hembras y 219 gr para el caso de los machos. Un lote de 45 reproductores de Bocachico $P$. reticulatus, 15 hembras y 30 machos fueron seleccionados para realizar la caracterización Morfológica y Meristica. Para estos ítems se tomaron las siguientes variables:

VARIABLES MORFOMETRICAS. Las medidas morfométricas fueron tomadas con un calibrador Vernier $(0.1 \mathrm{~mm})$, excepto para la longitud total y estándar que se midió con un ictiómetro convencional.

Longitud total- Longitud estándar- Ancho del cuerpo- Altura de la cabeza- Longitud de la cabeza- Longitud pre-dorsal

Longitud pre-anal- Número de escamas sobre la línea lateral.

Los reproductores de la especie Bocachico ( $P$. reticulatus) mostraron condiciones óptimas de maduración en los períodos de lluvias. Las condiciones físico-químicas del agua para el proceso se mantuvieron estables, se registró una temperatura promedio $28.88{ }^{\circ} \mathrm{C}$, un $\mathrm{pH}$ de 8.27 y niveles de amonio promedio de 0.27 .

Para la estandarización de la dosis se tomó guía las dosis referenciadas por Cordero C Argemiro (2003) para la especie Prochilodus magdalenae, teniendo en cuenta las condiciones de temperatura, $\mathrm{pH}$ y OD, que prevalecen para la zona.

Se tomaron las siguientes variables reproductivas:

- Edad Maduración sexual

- Número de ovas por hembra

- Horas de incubación

- Temperatura de incubaciónTiempo de desove

\section{RESULTADOS}

Los resultados para la evaluación morfo métrica se muestran en la tabla 1. 
Tabla 2. Parámetros morfo métricos en

\begin{tabular}{|c|c|c|c|c|c|c|c|c|c|}
\hline Machos & $\begin{array}{l}\text { Edad/ } \\
\text { meses }\end{array}$ & Long/total & Long/standard & $\begin{array}{l}\text { No. De } \\
\text { escamas }\end{array}$ & $\begin{array}{l}\text { Ancho } \\
\text { del } \\
\text { cuerpo }\end{array}$ & $\begin{array}{l}\text { Altura } \\
\text { de la } \\
\text { cabeza }\end{array}$ & $\begin{array}{l}\text { Long } \\
\text { de la } \\
\text { cabeza }\end{array}$ & $\begin{array}{l}\text { Long } \\
\text { /predorsal }\end{array}$ & Long/preanal \\
\hline Media & 8.00 & 18.51 & 13.50 & 40.98 & 2.52 & 3.00 & 3.21 & 3.00 & 9.57 \\
\hline Desv St & 0.0000 & 0.5500 & 0.6000 & 1.0000 & 0.6051 & 0.0000 & 0.6150 & 0.000 & 0.3413 \\
\hline $\begin{array}{l}\text { V } \\
\text { Mínimo }\end{array}$ & 8.0 & 18.4 & 12.0 & 40.0 & 2.4 & 3.0 & 3.0 & 2.90 & 7.32 \\
\hline $\begin{array}{l}\text { V } \\
\text { Máximo }\end{array}$ & 8.0 & 18.6 & 13.9 & 41.0 & 2.8 & 3.0 & 3.3 & 3.20 & 9.8 \\
\hline
\end{tabular}

reproductores machos (peso promedio $210 \mathrm{~g}$ )

Tabla 1. Parámetros morfo métricos de los reproductores hembras (peso promedio 250 \begin{tabular}{l|} 
Hembr \\
$\mathbf{a}$
\end{tabular}
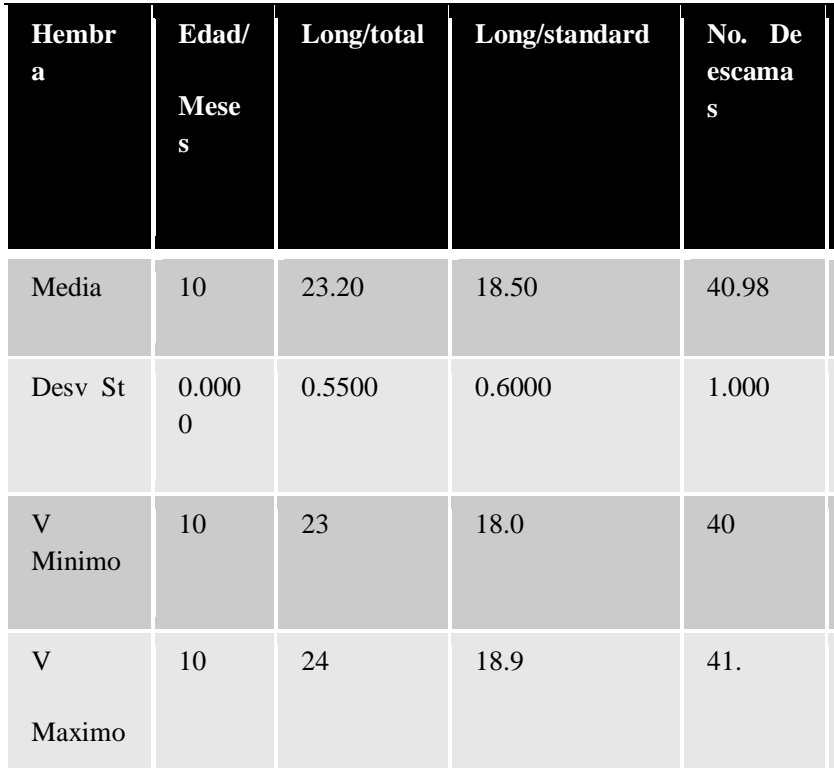

g)

Fuente: Elaboración propia
Las hembras de $P$. reticulatus presentan mayor desarrollo corporal que los machos a 
- Edad Maduración sexual

- Número de ovas por hembra
- Horas de incubación

- Temperatura de incubación

- Tiempo de desove

Tabla 3. Parámetros reproductivos: Edad 10 meses - peso promedio $250 \mathrm{~g}$.
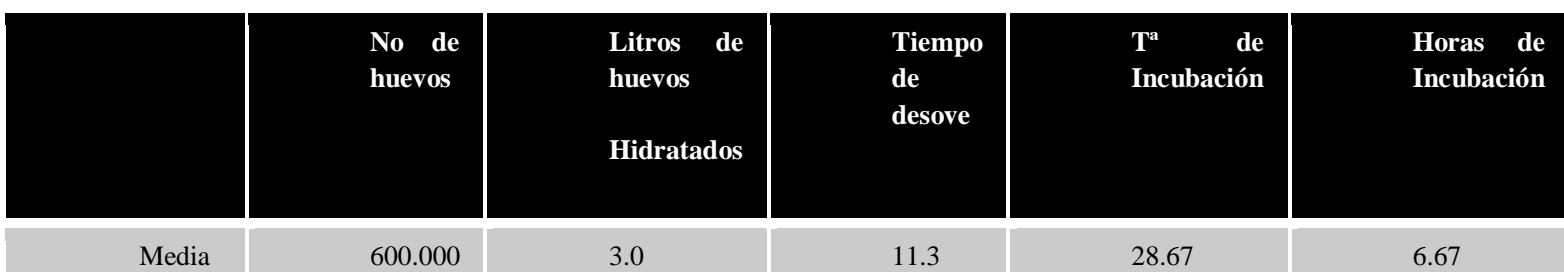

Des St

$$
600.000
$$

0.0000

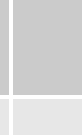

3.0

0.0000

Mínimo

Valor

Máximo
580.000

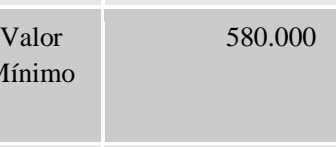

650.000

3.0

3.0
11.3

1.1500

10.00

12.0
28.67

1.1500

28.0

28.7

8.0

Fuente: Elaboración propia

Las variables reproductivas muestran un comportamiento eficiente en la reproducción, un número de ovas promedio de 600 es lo esperado para esta especie, aunque él \% de fertilización de $62 \%$ puede considerarse bajo, se encuentran en el rango esperado para las especies en cautiverio.

\section{PARÁMETROS DE PRODUCCIÓN}

La siguiente tabla permite consolidar los parámetros reproductivos y productivos para la Estación Piscícola San Pablo.

Tabla 4. Parámetros reproductivos y productivos para la Estación Piscícola San Pablo.

\begin{tabular}{|c|c|c|}
\hline PARÁMETRO & CANTIDAD & UNIDAD \\
\hline $\begin{array}{l}\text { Peso de reproductores } \\
\text { hembras }\end{array}$ & 341 & $\mathrm{Gr}$ \\
\hline $\begin{array}{l}\text { Peso de reproductores } \\
\text { machos }\end{array}$ & 219 & $\mathrm{Gr}$ \\
\hline No maduraciones por año & 2 & \\
\hline $\begin{array}{ll}\text { Dosificación } & \text { hormonal } \\
\text { Hembra (EPC) } & \end{array}$ & 6 & $\mathrm{mg} / \mathrm{kg}$ de $\mathrm{PV}$ \\
\hline $\begin{array}{l}\text { Dosificación hormonal } \\
\text { Hembra (EPC) }\end{array}$ & 4 & $\mathrm{mg} / \mathrm{kg}$ de $\mathrm{PV}$ \\
\hline Proporción hembra: macho & $1: 2$ & $\begin{array}{l}\text { Hembra: } \\
\text { Macho }\end{array}$ \\
\hline Presencia de desove & $152-176$ & Horas/grado \\
\hline Duración de desove & $4-6$ & Horas \\
\hline Incubación & 12 & Horas \\
\hline $\begin{array}{l}\text { Temperatura promedio al } \\
\text { desove }\end{array}$ & 27.1 & ${ }^{\circ} \mathrm{C}$ \\
\hline $\begin{array}{l}\text { Temperatura promedio de } \\
\text { incubación }\end{array}$ & 24.7 & ${ }^{\circ} \mathrm{C}$ \\
\hline $\begin{array}{llll}\text { Litros de } & \text { ovas } / \mathrm{kg} & \text { de } \\
\text { hembra } & & & \end{array}$ & 8.8 & $\mathrm{Lt}$ \\
\hline
\end{tabular}

\section{CONCLUSIONES.}

$P$. reticulatus, debe ser considerado uno de los recursos icticos mas importantes de la 
zona del Catatumbo, es una especie con excelente comportamiento reproductivo en cautiverio, responde de manera eficiente a los protocolos de reproducción inducida y sigue siendo una de las especies de mayor demanda por los consumidores, el aporte que la Universidad hace mediante la estandarización de la técnica de reproducción de esta especie, permitirá a todas las entidades y piscicultores de la zona, alcanzar resultados eficientes $y$ eficaces para mantener este recurso para las generaciones venideras.

\section{FINANCIACIÓN}

Agradecimiento a las entidades y personas que permitieron que la Estación Piscícola de San Pablo, fuera un referente para la zona del Catatumbo en el área de la producción piscícola, a los productores de la zona y de la provincia de Ocaña.

\section{BIBLIOGRAFÍA}

BARBARINO, A., D. TAPHORN Y K. O. WINEMILLER. 1998. Ecology of the coporo, Prochilodus mariae (Characiformes, Prochilodontidae), and status of annual migrations in western Venezuela. Environmental Biology of Fishes 53: 33-46.

CORDERO A., PERTUZ B, V; SOLANO G, J. 2003. Reproduccion Inducida del Bocachico (Prochilodus magdalenae Steindachner, 1878 con Ovaprim. Rev MVZ Cordoba, 8 (2): 335

FERNÁNDEZ-YÉPEZ, A. 1972. Análisis ictiológico del Complejo Hidrográfico (04) "Río Yaracuy”. Dirección de Obras Hidráulicas, Ministerio de Obras Públicas. República de Venezuela. 67 pp.

GÈRY, J. 1977. Characoids of the world. TFH Publications. Neptune City, New Jersey. 672 pp.

MAGO-LECCIA, F. 1968. Notas sobre los peces del río Guaire. Pp. 227-256. En: Estudio de Caracas. Ecología Vegetal y Fauna. vol. 1. Ediciones de la Biblioteca UCV. Caracas.

MARRERO, C. Y A. MACHADOALLINSON. 1990. Inventario y notas ecológicas de los peces de los ríos Panaquire, Urba y Yaguapa (cuenca del río Tuy) Edo. Miranda, Venezuela. Biollania 7: 55-82.

RODRÍGUEZ， J. P. 2001. La amenaza de las especies exóticas para la conservación de la biodiversidad suramericana. Interciencia 26(10): 479-483.

SNYDER, D. E. 1983. Fish Eggs and Larvae. $\quad$ Pp 165-198. En: Fisheries Techniques (Nielsen, L. A. and D. L. Johnson) (Eds.), American Fisheries Society, Maryland.

TAPHORN, D. C. 1992. The Characiform fishes of the Apure River drainage, Venezuela. Biollania Edición Especial 4: 1-537. Mem. Fund. La Salle de Cienc. Nat. 163137 


\section{ANEXOS}

Anexo 1. Protocolo para la reproducción y producción de alevinos de Bocachico

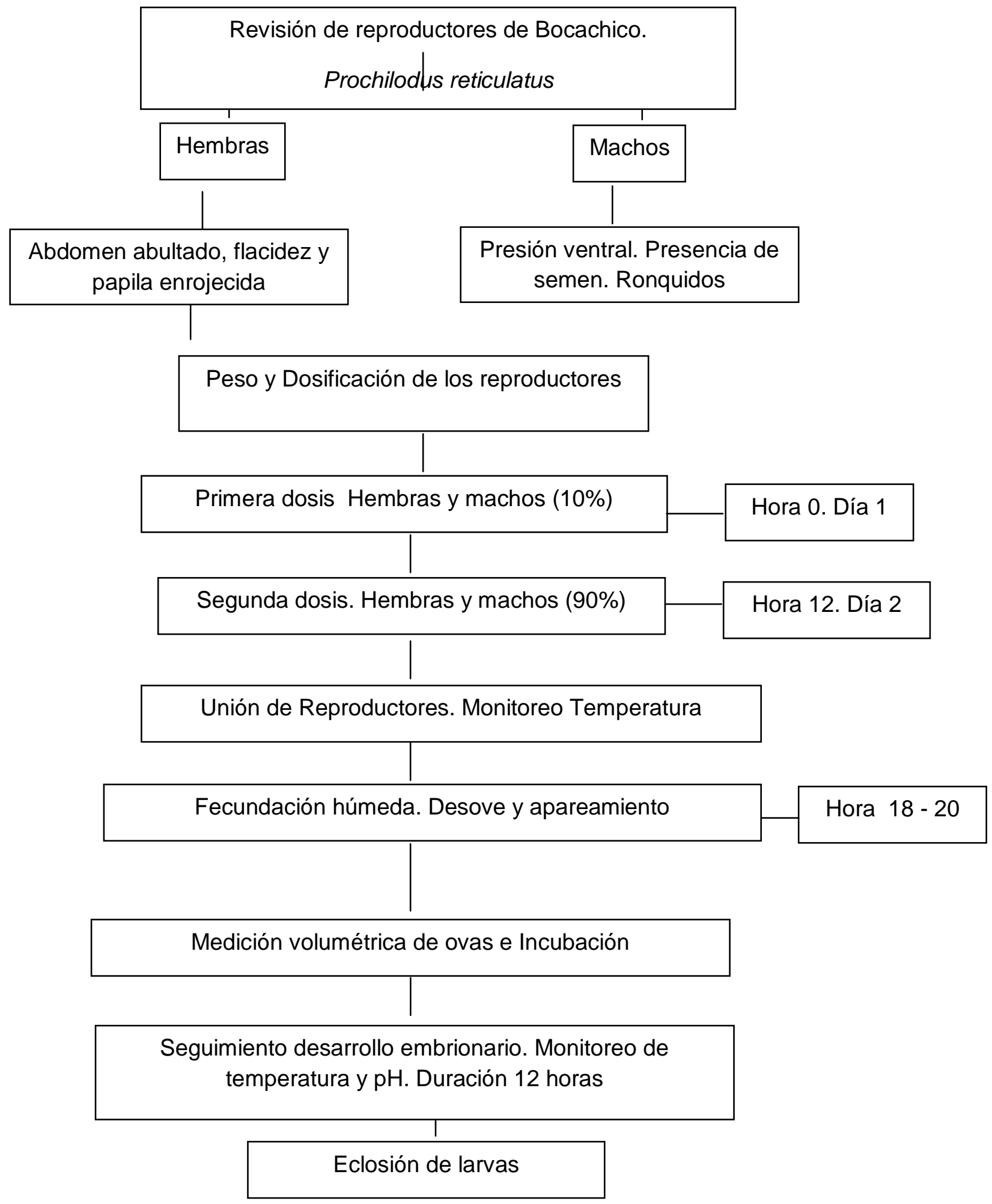

